\title{
Role of transient receptor potential and pannexin channels in cigarette smoke-triggered ATP release in the lung
}

\author{
Matthew Baxter, Suffwan Eltom, Bilel Dekkak, Liang Yew-Booth, Eric D Dubuis, \\ Sarah A Maher, Maria G Belvisi, Mark A Birrell
}

- Additional material is published online only. To view please visit the journal online (http://dx.doi.org/10.1136/ thoraxjnl-2014-205467).

Respiratory Pharmacology, Faculty of Medicine, National Heart and Lung Institute, Imperial College London, London, UK

\section{Correspondence to} Dr Mark Birrell, Respiratory Pharmacology, Faculty of Medicine, National Heart and Lung Institute, Imperial College London, Exhibition Road, London, SW7 2AZ, UK: m.birrell@imperial.ac.uk

Received 25 March 2014 Revised 4 August 2014

Accepted 13 September 2014 Published Online First 9 October 2014

\section{CrossMark}

To cite: Baxter $\mathrm{M}$, Eltom $\mathrm{S}$, Dekkak B, et al. Thorax 2014:69:1080-1089.

\section{ABSTRACT}

Background COPD is an inflammatory disease usually associated with cigarette smoking (CS) with an increasing global prevalence and no effective medication. Extracellular ATP is increased in the COPD affected lung and may play a key role in driving CS-induced airway inflammation, but the mechanism involved in ATP release has eluded researchers. Recently, the transient receptor potential (TRP) and pannexin-1 channels have been suggested to play a role in other experimental paradigms. Thus, the aim of this work is to investigate if these channels are involved in CSinduced ATP release in the lung.

Methods Primary human cells were exposed to CS and extracellular ATP levels measured. Mice were exposed to mainstream CS and airway inflammation assessed. TRPV1/ 4 mRNA expression was assessed in human lung parenchyma.

Results CS exposure caused a dose-related increase in ATP from primary airway bronchial epithelial cells. This was attenuated by blockers of TRPV1, TRPV4 and pannexin-1 channels. Parallel data was obtained using murine acute CS-driven model systems. Finally, TRPV1/4 mRNA expression was increased in lung tissue samples from patients with COPD.

Conclusions Extracellular ATP is increased in the COPD affected lung and may play a key role in driving disease pathophysiology. These experiments uncover a novel mechanism which may be responsible for CS-induced ATP release. These findings highlight novel targets that could lead to the development of medicine to treat this devastating disease.

\section{INTRODUCTION}

COPD is an inflammatory airway disease usually associated with cigarette smoking (CS) with an increasing global prevalence and no effective medication. ${ }^{1-3}$ The Global Initiative for Chronic Obstructive Lung Disease guidelines state that COPD is characterised by airflow limitation that is not fully reversible and is attributed to small airways disease and parenchymal destruction. It is a steroid-resistant, inflammatory disease characterised by the infiltration of leucocytes including neutrophils, macrophages and CD8 ${ }^{+}$T-cells. ${ }^{4}{ }^{5}$ Increased concentrations of many proinflammatory mediators are also observed including the caspase- 1 related cytokines, IL-1 $\beta$ and IL-18, the levels of which are found to correlate with disease severity. ${ }^{6} \quad 7$ Caspase- 1 activation is thought to be regulated by

\section{Key messages}

What is the key question?

- COPD is an airway disease with an increasing global prevalence but no effective medication. Understanding the disease pathogenesis would be a major step towards developing new therapies.

What is the bottom line?

- This study reveals for the first time the mechanism that leads to the increased ATP levels and the associated steroid-resistant inflammation observed in the airways of patients with COPD.

Why read on?

- These data highlight novel targets which can be exploited for the development of effective therapeutics for the treatment of COPD.

the NLRP3 inflammasome, and one of the key activation mechanisms is through extracellular ATP (eATP)-dependent stimulation of the $\mathrm{P}_{2} \mathrm{X}_{7}$ receptor. $^{8-10}$ Interestingly, increased levels of eATP have been reported in vitro and in vivo models of cigarette smoke-induced inflammation and in the lungs of patients with COPD. ${ }^{11-14}$ The relevance of this pathway to the pathogenesis of COPD is supported by findings that $\mathrm{P}_{2} \mathrm{X}_{7}$ receptor and caspase- 1 activation are central to the development of CS-induced airway inflammation in murine models. ${ }^{15-17}$

eATP is thought to be stored in the cytosol, but the mechanism by which it is released can depend on the cell/tissue type, the environment and the stimulus. ${ }^{18-21}$ Recent evidence has suggested a role for the pannexin-1 channel/pore in the mechanism of ATP release. ${ }^{22-27}$ Furthermore, transient receptor potential (TRP) channels have been suggested to play a pivotal role in the release of eATP and are associated with opening the pannexin-1 channel. ${ }^{182728}$ Of the TRP family, TRPV4 has been shown to be present in the airway and involved in the release of hypotonicity-induced eATP. ${ }^{27} 2930$ Endogenous TRPV1 activators (e.g. lipoxygenase products and mediators of the Protein Kinase-A, Protein Kinase- $\mathrm{C}$ and Phospholipase- $\mathrm{C}$ pathways) are known to be increased in the COPD affected 

lungs of patients with COPD as measured in exhaled breath condensate and this is also a trigger for TRPV1 activation. ${ }^{31}$ TRPA1, has also been implicated in COPD-associated disease pathologies. Indeed, Trpa $1^{-1-}$ mice were found to be protected against CS-mediated plasma protein extravasation and had reduced levels of the murine neutrophil chemoattractant keratinocyte chemoattractant (KC). ${ }^{32}$ Furthermore, TRPA1 is known to be activated by some of the key inflammatory components of CS, including acrolein and crotonaldehyde. ${ }^{33}$

Therefore, in view of the evidence presented above, we hypothesised that CS exposure induces ATP release via activation of specific TRP channels and the subsequent opening of the pannexin-1 pore. To test this hypothesis, we confirmed that CS exposure increased ATP levels in lavage in a murine model and that these levels were unchanged in $\mathrm{P}_{2} \mathrm{X}_{7}$ receptor-deficient mice that were protected from the CS-induced neutrophilia. This suggested that in our system the $\mathrm{P}_{2} \mathrm{X}_{7}$ receptor was not involved in the eATP release and that the signal was not from the recruited neutrophils but probably from cells present in the airway i.e. alveolar macrophages (AM) and human bronchial epithelial cells (HBEC). To study the mechanism responsible for the CS-induced release of ATP, we used primary human cellbased assays (AMs and HBECs) and pharmacological tools to investigate the role of TRPV1, TRPV4 and TRPA1 and pannexin-1. This was paralleled in our murine model using Trpv1, Trpv4, Trpa1 and pannexin-1 gene knockout (KO) mice. Finally, we measured the expression of the TRP channels in disease-relevant lung tissue harvested from patients with COPD to provide convincing data implicating these ion channels in the pathophysiology of COPD and highlighting them as novel targets for therapeutic intervention.

\section{MATERIALS AND METHODS \\ Cell-based model system}

CS bubbled medium (CSM) was prepared as described previously $^{34}$ and added to HBECs (Lonza, UK) or human AMs harvested as previously described. ${ }^{34}$ Cell viability was assessed using MTT. ATP levels were assessed using an ATPlite assay (Perkin Elmer, Cambridge, UK). Osmolarity and $\mathrm{pH}$ was assessed using an Osmomat 030 osmometer (Gonotec, Germany) and $\mathrm{pHep} 4 \mathrm{pH}$ meter (Hanna Instruments, UK), respectively. In some experiments, cells were pretreated with TRPV1, TRPV4 or TRPA1 antagonists (JNJ-17203212 (Sigma, UK), HC 067047 (Peakdale Molecular, UK) and HC 030031 (Chembridge, USA)), respectively, or agonists capsaicin (Sigma, UK), GSK 1016790a (Sigma, UK) and acrolein (Sigma, UK), respectively. In separate experiments cells were pretreated with specific pannexin antagonists carbenoxolone and probenecid or the connexin inhibitor flufenamic acid (all from Sigma, UK). Concentrations used were based on previous studies. ${ }^{24} 2735$

\section{Measurement of mRNA expression}

Quantitative RT-PCR was used to measure mRNA from human lung samples and cell populations using TaqMan reagents and protocols (Applied Biosystems) as previously described. ${ }^{36}$ Human donor and recipient lung tissue samples, surplus to clinical requirements, were obtained from a transplant programme supported by the NIHR Respiratory Disease Biomedical Research Unit at the Royal Brompton and Harefield NHS Foundation Trust and Imperial College London. Informed written consent and ethical approval was obtained (Ethic Number: REC 09/H0708/72, active from Dec 2009). See table 1 for demographic details. lung. Furthermore, low $\mathrm{pH}$ conditions often manifest in the

\section{In vivo studies}

All in vivo protocols were approved by a local ethical review process and strictly adhered to the Animals (Scientific Procedures) Act 1986 UK Home Office guidelines (Project Licence number: 70/7212, active from May 2012). Male wildtype $\mathrm{C} 57 \mathrm{BL} / 6$ were bred inhouse alongside the genetic lines. Mice were either kind gifts or purchased from Jackson labs, USA, $\left(P 2 X_{7}\right.$-Professor Jean Kanellopoulos (Université Paris-Sud, France), Trpv1 and Trpa1-Jackson labs; Trpv4Riken Bioresource Centre, (Japan) ${ }^{37}{ }^{38}$ and Pannexin-1-Dr Vishva Dixit, (Genentech, USA)).

CS or lipopolysaccharide (LPS) exposure protocols have been described previously. ${ }^{15}$ Briefly, mice were exposed to CS (1 h, twice a day for 3 days-acute model) or LPS (aerosol of $1 \mathrm{mg} / \mathrm{mL}$ for $30 \mathrm{~min}$ ) and the lungs lavaged $24 \mathrm{~h}$ or $6 \mathrm{~h}$ later, respectively. Bronchoalveolar lavage fluid (BALF) interleukin (IL)-1ß (ELISA, assay range reported as $15.6-1000 \mathrm{pg} / \mathrm{mL}$; to measure lower levels it is important to freshly prepare the assay plates) neutrophil and ATP levels (no increase detected in the LPS model) were assessed as previously described. ${ }^{15}$ A parallel subchronic model was also performed where the wild-type or $\mathrm{KO}$ mice were exposed to CS for 14 days.

\section{Data analysis}

Data are expressed as mean \pm SEM of $n$ observations. Statistical significance was determined using either single or multiple comparisons (specific tests used are described in the figure legends), using GraphPad Prism 5 software. A p value $<0.05$ was taken as significant and all treatments were compared with the appropriate control group.

\section{Online supplementary material}

The online supplementary material section contains the data from characterising the impact of smoke on cell/medium in vitro, profiling the TRPV1, TRPV4 and pannexin-1 KO mice in the LPS-driven model of airway inflammation. Additionally, it contains the data from profiling the TRPA1 KO mice in the smoke and LPS model systems.

\section{RESULTS}

$\mathrm{P}_{2} \mathrm{X}_{7}$ receptor and neutrophils are not involved in acute CS mediated ATP release in vivo

Wild-type and $\mathrm{P} 2 \mathrm{X}_{7}^{-1-}$ mice were concurrently exposed to CS twice a day for three consecutive days. There was a significant increase in ATP, IL-1 $\beta$ and neutrophils in the BALF in CS-challenged wild-type mice $24 \mathrm{~h}$ after the last exposure (figure 1). $\mathrm{P} 2 \mathrm{X}_{7}^{-1-}$ mice exhibited significantly reduced levels of IL-1 $\beta$ and neutrophilia compared with the wild-type consistent with previously published data ${ }^{15}{ }^{16}$ (figures $1 \mathrm{~B}, \mathrm{C}$ ). There was, however, no reduction in the levels of BALF ATP in CS-exposed $\mathrm{P}_{2} \mathrm{X}_{7}^{-/-}$mice compared with the wild-type controls. This

Table 1 Details on the donor/recipient

\begin{tabular}{llc}
\hline Disease group & Age (years) & Sex (M/F) \\
\hline Non-smoking donor & $27-72$ & $3 / 9$ \\
Smoking donor & $22-62$ & $7 / 3$ \\
Emphysema & $49-69$ & $17 / 8$ \\
Non-smoking donor & $27-72$ & $3 / 9$ \\
Smoking donor & $22-62$ & $7 / 3$ \\
Emphysema & $49-69$ & $17 / 8$ \\
\hline
\end{tabular}

Baxter M, et al. Thorax 2014;69:1080-1089. doi:10.1136/thoraxjnl-2014-205467 
Figure 1 The role of $\mathrm{P}_{2} \mathrm{X}_{7}$ in cigarette smoking (CS)-induced murine airway inflammation. $\mathrm{P}_{2} \mathrm{X}_{7}^{-1-}$ mice were exposed to $\mathrm{CS}$ or room air (control) twice daily for 3 consecutive days alongside wild-type controls. Bronchoalveolar lavage fluid was collected $24 \mathrm{~h}$ after the last exposure for measurement of neutrophil (C), interleukin (IL)-1 $\beta$ (B) and ATP (A) levels. Data are represented as mean \pm SEM for $n=8$ animals in each group. Statistical significance was determined using Mann-Whitney U test. \#p $<0.05$, denoting a significant difference between the smoke-exposed and air-exposed wild-type groups; ${ }^{*} \mathrm{p}<0.05$, denoting a significant difference between the smoke-exposed knock-outs (KO) and wild-types.
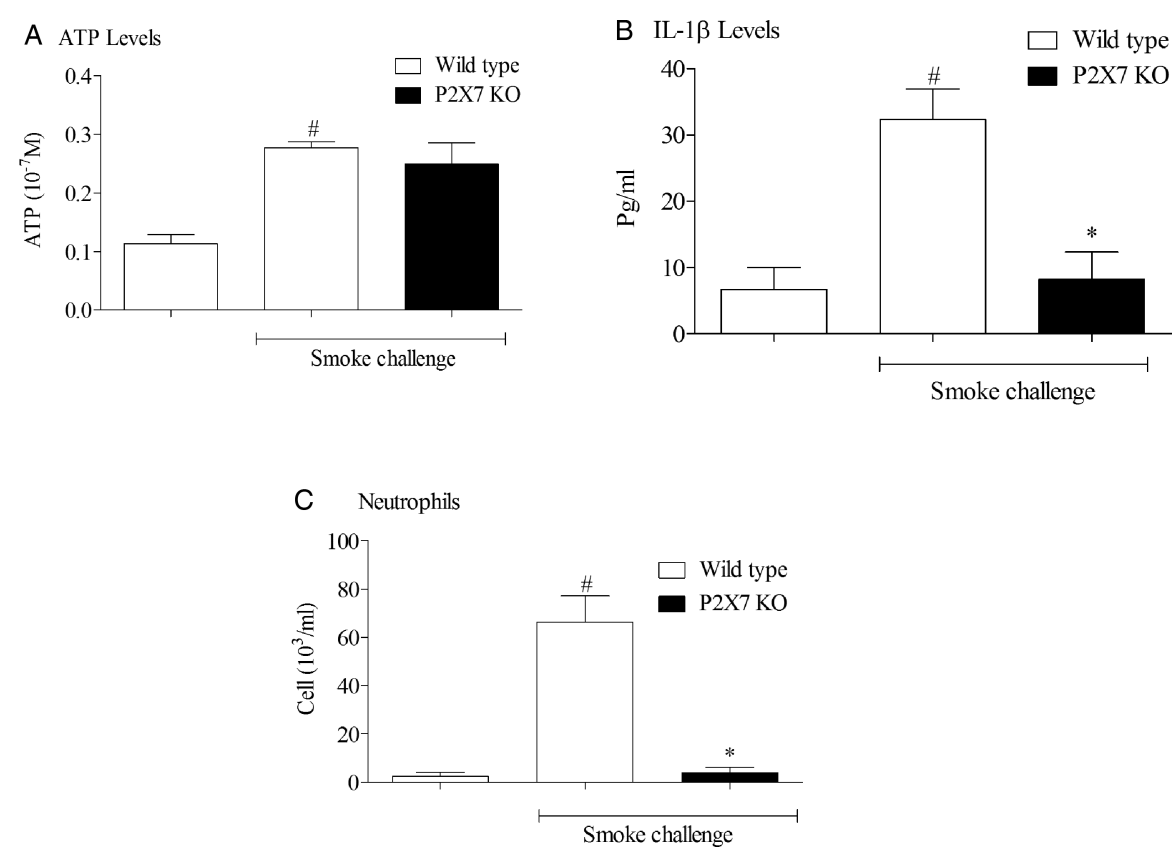

indicates that the ATP release is independent of the $\mathrm{P} 2 \mathrm{X}_{7}$ receptor and that it is not released from the recruited neutrophils. This also suggests that CS-induced ATP is upstream of the P2 $\mathrm{X}_{7}$-inflammasome-IL-1 $\beta / \mathrm{IL}-18$ axis and is released from structural and/or resident cells in the airway, possibly AMs and bronchiolar epithelial cells.

\section{Development and characterisation of CS-induced ATP release in vitro}

To begin to investigate the mechanism responsible for CS-induced ATP release we used cell-based assays. We selected airway-relevant primary cells (HBECs and AMs) and exposed them to CSM. CSM caused a dose-related release of ATP over time in both cell types (figure 2A, C). CSM (2.5\%) was chosen for further analysis. CSM did not cause any cell death or alter the $\mathrm{pH}$ or osmolarity of the culture medium (see online supplementary figure S1). Analysis of the mRNA levels of the TRP channels under investigation in the two cell types showed that both cell types expressed significant levels of TRPV1 and TRPV4 mRNA but the levels of TRPA1 were below the detection limit of the assay in AMs and detected only at very low levels in HBECs (figure 2D).

\section{Role of TRPV1, TRPV4 and pannexin channels in CSM-mediated ATP release in vitro}

CSM-induced ATP release from HBECs was inhibited by a TRPV4 antagonist and, to a lesser extent, by a TRPV1 antagonist (figure 3). In parallel, the pannexin channel blockers
Figure 2 Characterisation of ATP release from $\mathrm{HBEC}$ and $\mathrm{AMs}$ in vitro. For concentration-response experiments (A and C), HBECs and AMs were stimulated with increasing concentrations $(0.312 \%-10 \%)$ of CSM or vehicle (normal medium). Cells were incubated for $3 \mathrm{~h}$ before removal of supernatant and measurement of ATP levels. For time-course experiments ( $B$ and C), cells were stimulated with CSM $(2.5 / 5 \%)$ or vehicle. Supernatants were removed from selected cell populations at various time-points $(1,3,6,24 \mathrm{~h})$ after stimulation for measurement of ATP. For both, concentration-response and time-course experiments, data are represented as mean $\pm S E M$ for $n=3-5$ donors. (D) TRPA1, V1 and V4 mRNA expression was measured in HBECs and AMs using TaqMan reagents and protocols. Data are expressed as mean \pm SEM expression levels relative to internal control (18S). TRP, transient receptor potential; $\mathrm{HBEC}$, human bronchial epithelial cells; AM, alveolar macrophages; CSM, cigarette smoking bubbled medium.

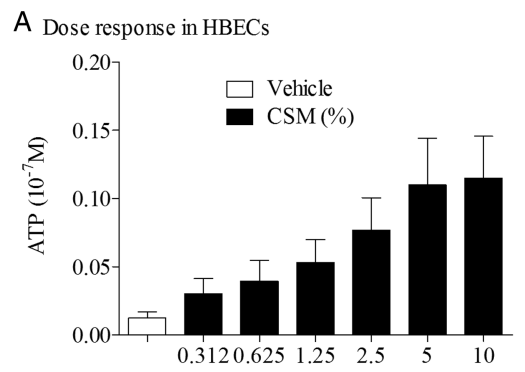

C

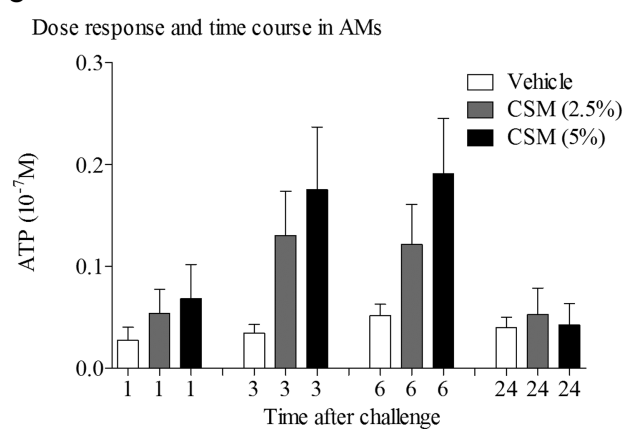

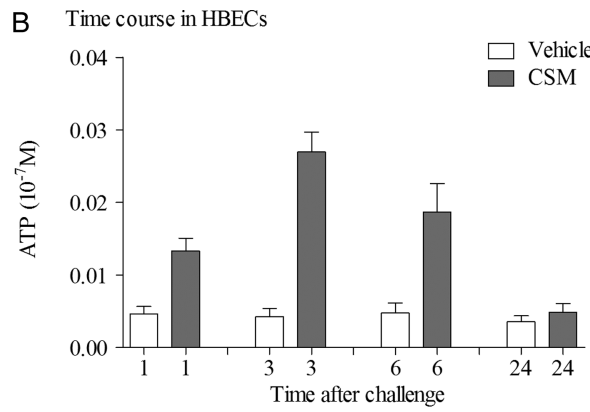

D

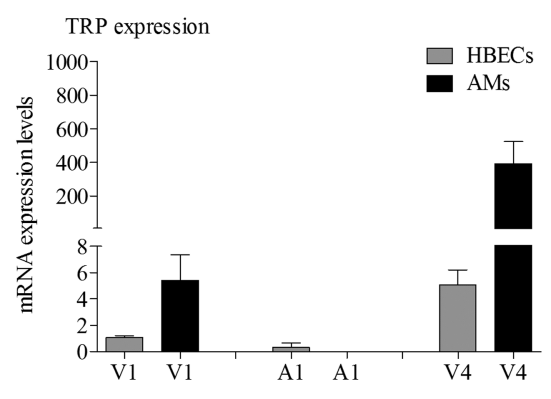


(carbenoxolone and probenecid), but not the connexin channel blocker (flufenamic acid) modulated the ATP levels (figure 3). To complement the antagonist data, we examined whether specific TRP channel agonists were able to stimulate ATP release directly in HBECs. A dose-dependent ATP release was observed using the TRPV1 agonist capsaicin (figure 4A) and the TRPV4 agonist HC-067047 (figure 4B), but there was no effect with the TRPA1 agonist acrolein (figure 4C). In summary, pharmacological data advocate a role for TRPV1 and TRPV4, but not TRPA1 in CSM-stimulated ATP release from HBECs via pannexin channels.

\section{Role of $\mathrm{P}_{2} \mathrm{X}_{7}$ in ATP and LPS-induced IL-1 $\beta$ release in vitro}

The above data, in combination with the published literature, ${ }^{15} 16$ suggests that inhaling smoke leads to the release of eATP via the activation of TRPV1/4 receptors and opening of pannexin-1 channels. ATP then acts on $\mathrm{P} 2 \mathrm{X}_{7}$ receptors to trigger the release of the inflammasome products IL-1 $\beta / \mathrm{IL}-18$. Indeed, Lucattelli et al, ${ }^{16}$ using irradiated mice, suggest that this response was dependent on $\mathrm{P}_{2} \mathrm{X}_{7}$ receptor activation on haemopoietic cells, and not structural cells. From this, we proposed that ATP could cause the release of IL- $1 \beta$ from our cell-based system. Thus, we profiled the effect of ATP, and a second known IL-1 $\beta$ release trigger (LPS-an innate stimulus), on IL-1 $\beta$ levels in culture HBECs and AMs. Time points and assay conditions were chosen from previous studies. ${ }^{15}{ }^{34}$ Neither ATP nor LPS triggered the release of IL-1 $\beta$ from HBECs but they did cause a concentration-induced release from AMs (figure 5). Consistent with these observations, we found significant expression levels of caspase 1 and $\mathrm{P}_{2} \mathrm{X}_{7}$ in AMs, but very low levels in HBECs $(163 \pm 42$ and $208 \pm 95$ vs $0.04 \pm 0.02$ and $3.7 \pm 0.7$ $2^{\text {deltaCT }} \times 10^{6}$, respectively). Finally, we found that blockade of the $\mathrm{P} 2 \mathrm{X}_{7}$ receptor resulted in a concentration-related decrease in ATP-induced IL-1 $\beta$ release but had no effect on the normal, defensive, innate response. Together, these data support the hypothesis that ATP can induce IL-1 $\beta$ from haemopoietic cells (AMs) via the $\mathrm{P} 2 \mathrm{X}_{7}$ receptor.

\section{Role of TRPV1, TRPV4 and pannexin-1 channels in CS-mediated ATP release and the inflammatory response in a murine model}

Trpv1 $1^{-/-}$and Trpv $4^{-/-}$mice were found to exhibit reduced levels of ATP, IL-1 $\beta$ and neutrophilia, in the BALF, after acute CS exposure (figures 6 and 7). Similarly, pannexin- $1^{-/-}$mice exhibited significantly reduced levels of BALF ATP and neutrophilia compared with wild-type mice after acute CS exposure, and no increase in IL-1 $\beta$ levels (figure 8). In this study, we observed an increase in BALF IL- $1 \alpha$ levels that were abolished in the pannexin-1 KOs (air/WT $=16.5 \pm 7.2$, air $/ \mathrm{KO}=18.4 \pm 4.5$, smoke/WT $=36.7 \pm 4.5, \quad$ smoke $/ \mathrm{KO}=17.6 \pm 4.0)$. Interestingly, Trpv1, Trpv4 or pannexin-1 knockouts were not compromised in their ability to mount an inflammatory response to an innate stimulus suggesting that they would not be immune compromised (see online supplementary figures S2, S3 and S4). TRPA1 deficient mice did not have an altered response profile, compared with wild-type controls, after either CS or LPS challenge (see online supplementary figure S5). In the subchronic model, the numbers of neutrophils in the $\operatorname{Trpv} 1^{-/-}$and $\operatorname{Trpv} 4^{-/-}$mice were found to be lower than the wild-type controls (figure 9).

Taken together, these data suggest that CS mediates inflammation in these murine in vivo models by activation of TRPV1 and TRPV4 (but not TRPA1), leading to ATP release via pannexin channels.

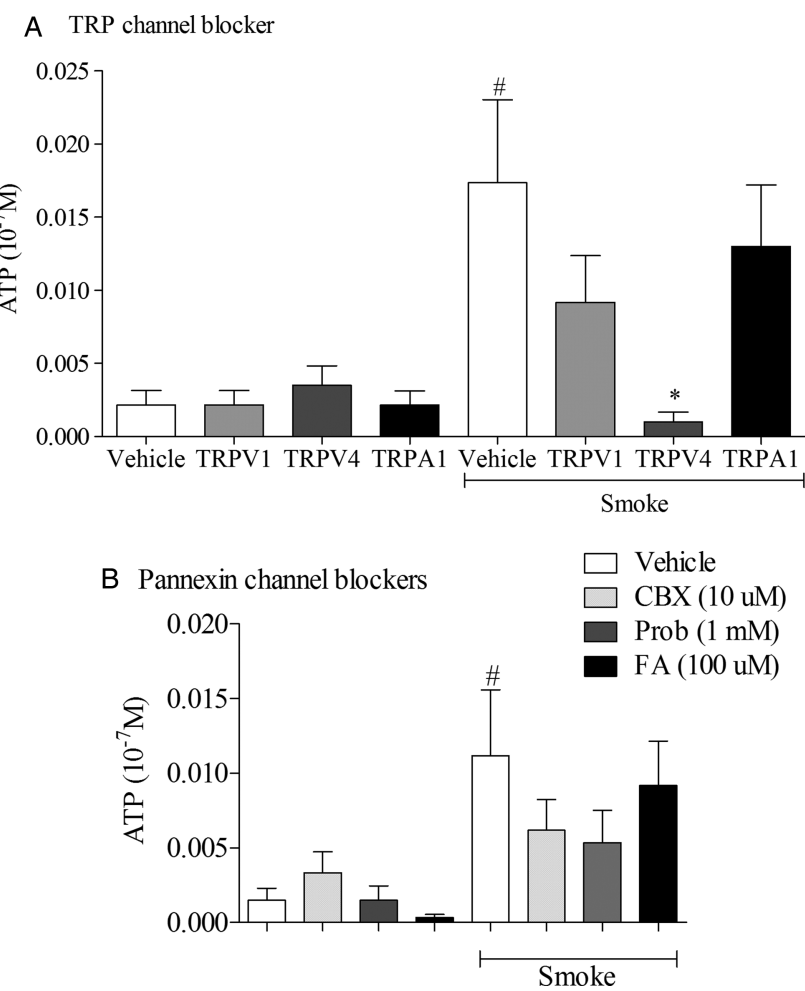

Figure 3 The effect of transient receptor potential (TRP) channel and pannexin channel antagonists on cigarette smoking bubbled medium (CSM) induced ATP release in vitro. (A) Human bronchial epithelial cells (HBECS) were preincubated with either TRP channel antagonist: HC-030031 (TRPA1, $100 \mu \mathrm{M})$, JNJ-17203212 (TRPV1, $10 \mu \mathrm{M})$, HC-067047 (TRPV4, $10 \mu \mathrm{M}$ ); or vehicle (dimethyl sulfoxide (DMSO), $0.1 \% \mathrm{v} / \mathrm{v})$. (B) HBECs were preincubated with either a pannexin or connexin antagonist or vehicle (DMSO, $0.1 \% \mathrm{v} / \mathrm{v}$ ). After preincubation with the respective antagonist or vehicle for $1 \mathrm{~h}$, cells were treated with $2.5 \%$ CSM or control medium and incubated for $3 \mathrm{~h}$ before removal of supernatants for measurement of ATP levels. Data are represented as mean \pm SEM for $n=3$ donors. $\# p<0.05$, denoting a significant difference between the CSM-treated and control-treated vehicle groups (using Mann-Whitney $U$ test); ${ }^{*} p<0.05$, denoting a significant difference between the respective CSM/antagonist group and the CSM/vehicle group (using one-way analysis of variance followed by Dunn's Multiple Comparison Test).

\section{Expression of TRPV1 and TRPV4 in patients with COPD}

To assess the translational merits of this murine in vivo data, we examined the expression levels of selected TRP channels in human lung parenchyma from healthy non-smokers, healthy smokers and patients with COPD (see table 1 for demographic details). TRPV1 and TRPV4 expression levels were significantly increased in patients with COPD compared with healthy non-smokers, but there was no difference between healthy smokers and healthy non-smokers (figure 10A, D). There was no difference in TRPV2 expression between any of the groups (figure 10B). TRPV3 expression was below the detection limits of the assay in all three groups (figure 10C). TRPA1 levels were below the assay's reliable detection limit.

While this data is encouraging, it is necessary to confirm these finding in samples harvested from a larger, well characterised patient group. Furthermore, the parenchyma was not sampled from specific anatomical positions for a truly accurate comparison. 
A HBECs - TRPV1

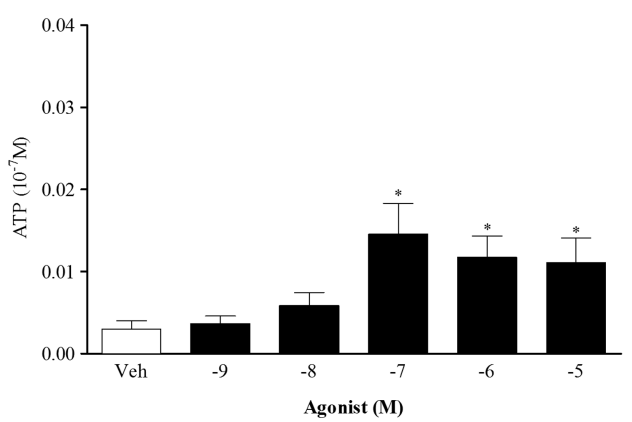

B HBECs - TRPV4

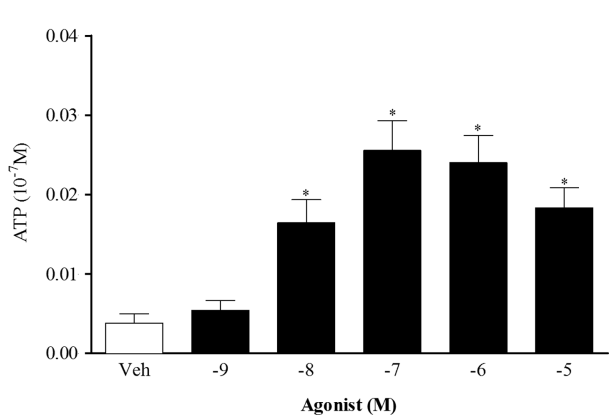

C HBECs - TRPA1

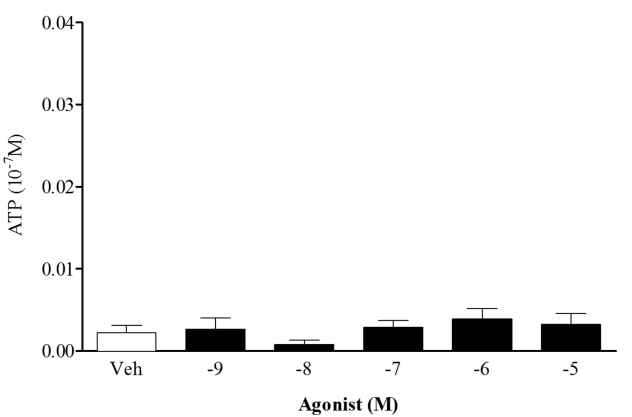

Figure 4 The effect of transient receptor potential (TRP) channel agonists on ATP release in vitro. Human bronchial epithelial cells (HBECS) were stimulated with increasing concentrations of agonist or vehicle. (A) TRPV1 agonist, capsaicin; (B) TRPV4 agonist, GSK 1016790A; (C) TRPA1 agonist, acrolein. Supernatants were collected $3 \mathrm{~h}$ after stimulation for assessment of ATP release. Data are expressed as mean $\pm S E M$ for $n=3$ donors; ${ }^{*} \mathrm{p}<0.05$, denoting a significant difference between the vehicle and agonist stimulation (using one-way analysis of variance followed by Dunn's Multiple Comparison Test).

\section{DISCUSSION}

Chronic exposure to CS is a key causative factor in the development of COPD. ${ }^{39}$ It is thought to result in a dysregulated inflammatory response in the airways characterised by high levels of leucocyte infiltration and inflammatory mediator release which drive disease progression and lung function decline. Elucidation of the pathways which mediate CS-induced inflammation is likely to uncover novel targets for the development of effective therapies. Patients with COPD exhibit increased levels of eATP, IL-1 $\beta$ and IL-18 in the BALF, as well as increased expression of $\mathrm{P}_{2} \mathrm{X}_{7}$ and caspase- 1 in leucocytes and lung tissue, and importantly, these markers are found to correlate with disease severity. ${ }^{6} 7{ }^{15}$ Murine in vivo models of CS-induced airway inflammation have also been shown to be dependent on the $\mathrm{P} 2 \mathrm{X}_{7}$-dependent activation of caspase- 1 to mediate the maturation of cytokines IL- $1 \beta$ and IL-18, indicating that these models have disease relevance. Pharmacological and/or genetic manipulation of ATP and these proteins have been shown to attenuate CS-induced neutrophilia in both acute and more chronic murine models, suggesting that modulation of this pathway could potentially reduce the steroid-resistant inflammation which drives the progression of COPD. ${ }^{15} 1640$ Despite this wealth of data indicating a role for ATP, the mechanism by which it is released after exposure to cigarette smoke has so far eluded researchers and, if identified, could reveal novel targets for the development of truly effective therapies.

The data presented herein strongly advocate a crucial role for TRPV1 and TRPV4 channels in the pannexin-1-mediated release of ATP in response to acute CS exposure. This was demonstrated in human primary cells using TRPV1, TRPV4 and pannexin-1-specific antagonists to block CSE-induced ATP release, as well as TRPV1 and TRPV4 agonists to directly elicit ATP release. The importance of this TRP channel pannexin-1 axis was recapitulated in vivo using colonies of $\operatorname{Trpv} 1^{-/-}$, Trpv $4^{-/}$, and pannexin- $1^{-/}$genetically deficient mice. Importantly, these mice exhibited reduced ATP levels after acute CS exposure, and they also exhibited attenuated IL-1 $\beta$ and neutrophil levels in BALF compared with wild-type, demonstrating that this pathway is integral to acute CS-induced inflammatory response, which is thought to drive the progression of COPD. These findings provide a novel insight into the role of these channels in the generation of acute CS-induced airway inflammation. By comparison, genetic ablation of the $\mathrm{P}_{2} \mathrm{X}_{7}$ receptor was found to protect CS-exposed mice against increased BALF IL-1 $\beta$ and neutrophilia, but did not alter ATP release. These observations indicate that ATP is released upstream of $\mathrm{P}_{2} \mathrm{X}_{7}$ receptor activation in this model and help to unravel the relationship between pannexin- 1 and the inflammasome. These data support the hypothesis that in this system, pannexin-1 stimulation results in inflammasome activation indirectly, via the release of eATP resulting in subsequent $\mathrm{P}_{2} \mathrm{X}_{7}$ activation. ${ }^{21}$ While these data in the acute and subchronic models are very exciting, further work in chronic models is required to fully elucidate the role of this axis in the pathogenesis of clinical COPD.

Evidence suggests that this inflammatory pathway is important in COPD, as translational data has shown increased levels of $\mathrm{P}_{2} \mathrm{X}_{7}$ expression and caspase-1 activation in CS-exposed wildtype mice and in patients with COPD. ${ }^{12}{ }^{15}$ Furthermore, the preliminary demonstration that TRPV1 and TRPV4 gene expression are also upregulated in lung tissue from patients with COPD suggests that these channels may be important for the CS-mediated release of eATP in this disease. It could also 


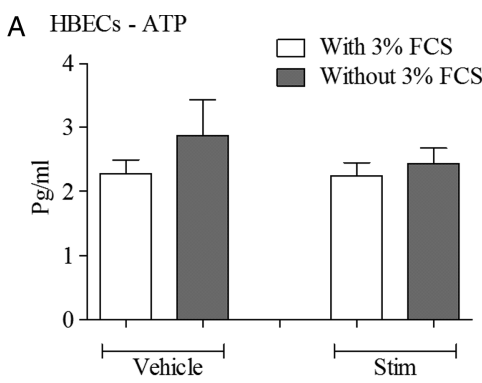

C

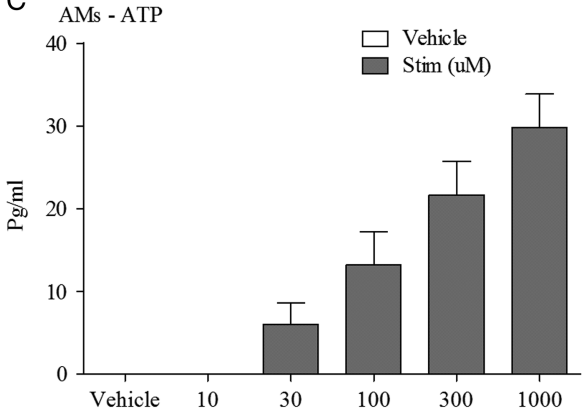

$\mathrm{E}$

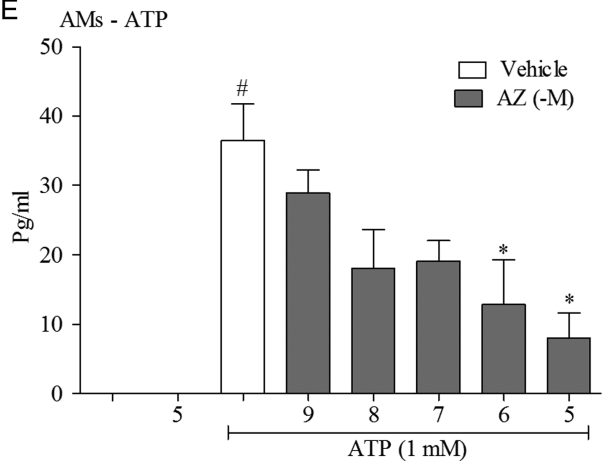

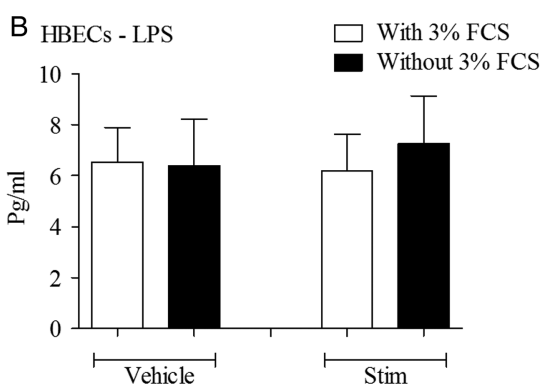
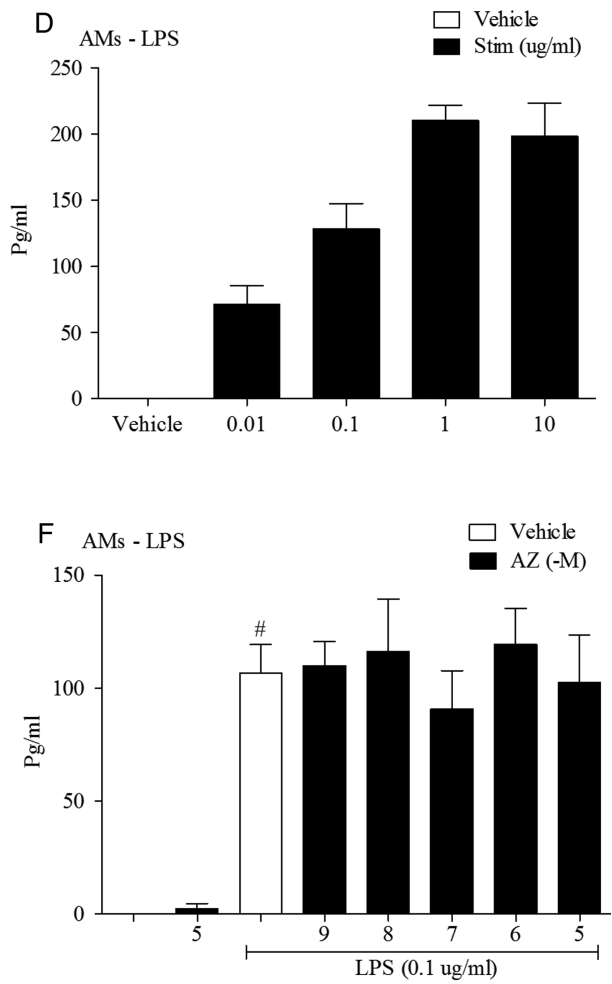

Figure 5 Characterisation of ATP-induced interleukin (IL)-1 $\beta$ release in vitro. A: Human bronchial epithelial cells (HBECS), cultured in medium with or without $3 \%$ fetal calf serum (FCS), were stimulated with ATP $(0.3 \mathrm{mM})$ or vehicle (phosphate buffered saline (PBS) $0.1 \%$ v/v). Supernatants were collected $24 \mathrm{~h}$ after stimulation for IL-1 $\beta$ measurement by ELISA. B: HBECs, cultured in medium with or without $3 \%$ FCS, were stimulated with lipopolysaccharide (LPS) $(0.1 \mu \mathrm{g} / \mathrm{mL}$ ) or vehicle (PBS, $0.1 \% \mathrm{v} / \mathrm{v})$. Supernatants were collected $24 \mathrm{~h}$ after stimulation for IL-1 $\beta$ measurement by ELISA. Data for $(A)$ and $(B)$ are represented as mean $\pm S E M$ for $n=3$ donors. (C) and (D): alveolar macrophages (AM) were stimulated with (C) increasing concentrations of ATP from 10 to $1000 \mu \mathrm{M}$ or (D) increasing concentrations of LPS from 0.01 to $10 \mu \mathrm{g} / \mathrm{mL}$. Supernatants were collected $24 \mathrm{~h}$ after stimulation for IL-1 $\beta$ measurement by ELISA. (E) and (F) AMs were preincubated with increasing concentrations of P2X7 antagonist (AZ-11645373) or vehicle (dimethyl sulfoxide, $0.1 \%$, v/v) for $1 \mathrm{~h}$ before stimulation with (E) either $1 \mathrm{mM}$ ATP or vehicle; or (D) either $0.1 \mu \mathrm{g} / \mathrm{mL} \mathrm{LPS}$ or vehicle. Cells were subsequently incubated for a further $24 \mathrm{~h}$ before collection of supernatants and measurement of IL-1 $\beta$. Data for (C), (D), (E) and (F) are represented as mean \pm SEM for $n=3$ donors. ( $E$ ) $\# p<0.05$, denoting a significant difference between the ATP/vehicle treated and vehicle/ vehicle-treated groups (using Mann-Whitney $\mathrm{U}$ test); ${ }^{*} \mathrm{p}<0.05$, denoting a significant difference between the ATP/vehicle-treated group and the respective ATP/antagonist-treated group (using one-way analysis of variance followed by Dunn's Multiple Comparison Test). (F): \#p $<0.05$, denoting a significant difference between the LPS/vehicle treated and vehicle/vehicle-treated groups (using Mann-Whitney U test).

provide some preliminary insight into COPD susceptibility and indicate why only a certain percentage of smokers develop COPD (i.e. increased channel expression means higher levels of ATP). Indeed, TRPV4 single nucleotide polymorphisms have been associated with COPD susceptibility in two separate studies. ${ }^{41} 42$

An interesting feature of this inflammatory pathway is the fact that release of eATP to activate the $\mathrm{P} 2 \mathrm{X}_{7}$-inflammasome-caspase- 1 axis facilitates the production of IL-1 $\beta$ and IL-18 without the need for transcriptional upregulation of the genes which encode these proinflammatory cytokines. Rather, there are substantial levels of the proforms of these cytokines constitutively present in the cytosol, for which caspase-1 facilitates the maturation to active, proinflammatory mediators. Indeed, previous investigation of murine models has demonstrated that IL-1 $\beta$ mRNA levels are not affected by CS exposure, despite significantly higher levels of IL-1 $\beta$ protein. ${ }^{15}$ This may, in part, explain the insensitivity of patients with COPD to glucocorticoids, as they are generally thought to exert their anti-inflammatory effects by modulation of inflammatory cytokines at the transcriptional level. Pharmacological intervention at the TRPV1/4-pannexin-1 axis may, therefore, prove to be an effective anti-inflammatory therapeutic strategy where glucocorticoids have failed. Furthermore, TRPV1, TRPV4 and pannexin-1 gene-deleted mice mounted a 
A

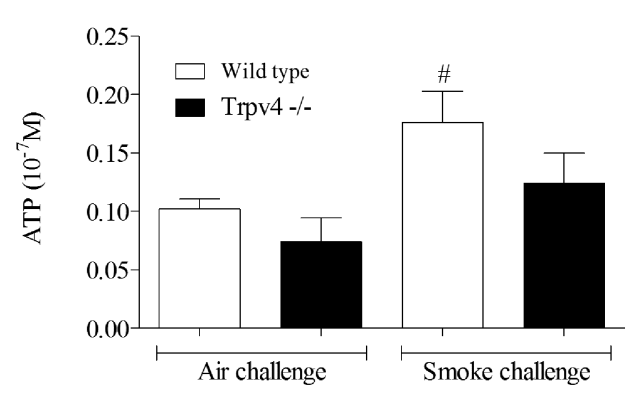

B BALF IL-1 $\beta$

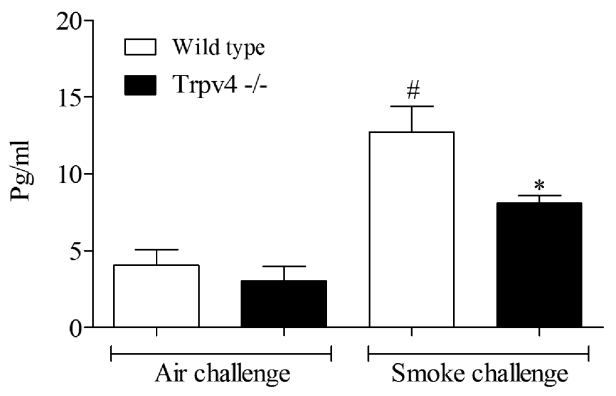

C BALF Neutrophilia

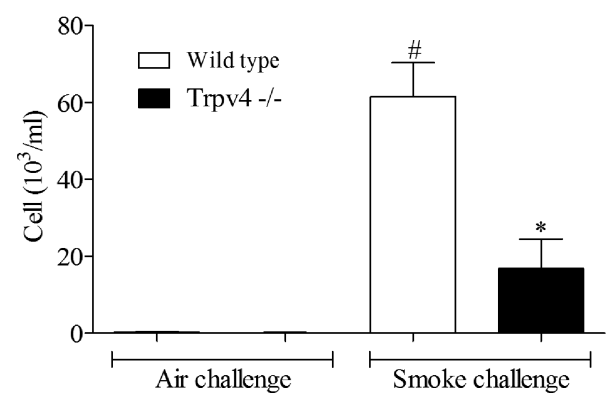

Figure 6 The role of TRPV4 in acute CS-induced murine airway inflammation. Trpv4 ${ }^{-l-}$ mice were exposed to CS or room air (control) twice daily for 3 consecutive days alongside wild-type controls. BALF was collected $24 \mathrm{~h}$ after the last exposure for measurement of neutrophil (C), IL-1 $\beta$ (B) and ATP (A) levels. Data are represented as mean \pm SEM for $n=8$ animals in each group. Statistical significance was determined using Mann-Whitney $U$ test. $\# p<0.05$, denoting a significant difference between the smoke-exposed and air-exposed wild-type groups; ${ }^{*} p<0.05$, denoting a significant difference between the smoke-exposed knock-outs and wild-types. TRP, transient receptor potential; CS, cigarette smoking; BALF, bronchoalveolar lavage fluid; IL-1 $\beta$, interleukin-1 $\beta$.

normal innate immune response to inhaled bacterial mimetic, LPS, suggesting that therapies that target this axis would not immunecompromise patients. To confirm this suggestion, further work is required with live bacteria and respiratory viruses. Somewhat surprisingly, TRPA1 did not play a role in CS-induced inflammation in either the in vivo or in vitro models even though it can be directly activated by acrolein, one of the key components of CS. One explanation could be that the cell types we studied did not express TRPA1.

A question remains regarding the mechanism by which CS activates TRPV1 and TRPV4 in these models. We initially hypothesised that TRPV1 and TRPV4 may be activated by low $\mathrm{pH}$, but
Figure 7 The role of TRPV1 in acute CS-induced murine airway inflammation. Trpv1 $1^{-1-}$ mice were exposed to CS or room air (control) twice daily for 3 consecutive days alongside wild-type controls. BALF was collected $24 \mathrm{~h}$ after the last exposure for measurement of neutrophil (C), IL-1 $\beta$ (B) and ATP (A) levels. Data are represented as mean \pm SEM for $n=8$ animals in each group. Statistical significance was determined using Mann-Whitney U test. \#p $<0.05$, denoting a significant difference between the smoke-exposed and air-exposed wild-type groups; ${ }^{*} p<0.05$, denoting a significant difference between the smoke-exposed knock-outs and wild-types. TRP, transient receptor potential; CS, cigarette smoking; BALF, bronchoalveolar lavage fluid; IL-1 $\beta$, interleukin-1 $\beta$.

$$
\text { A BALF ATP }
$$

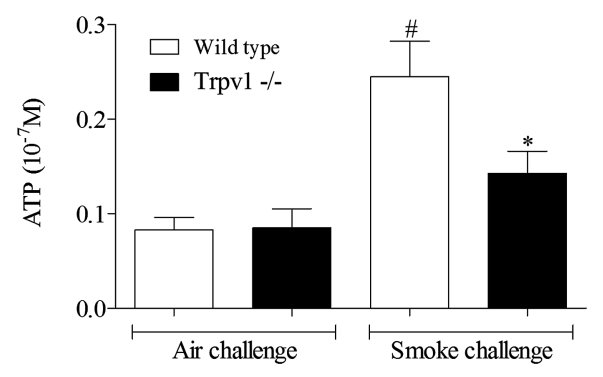

B BALF IL-1 $\beta$

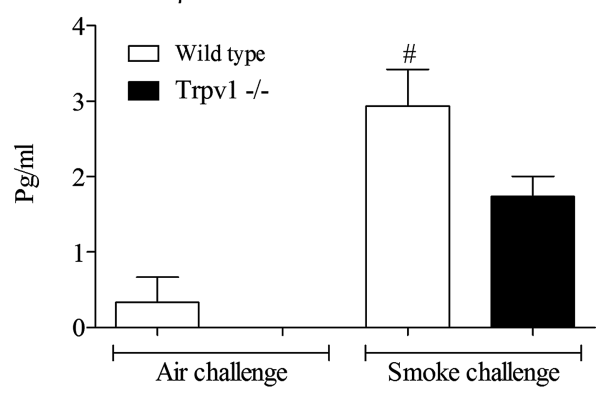

C BALF Neutrophilia

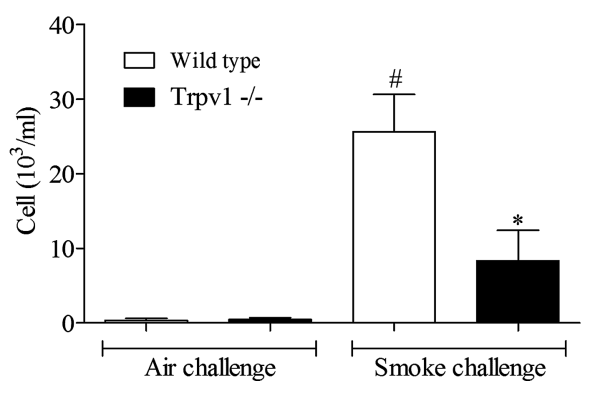


A BALF ATP

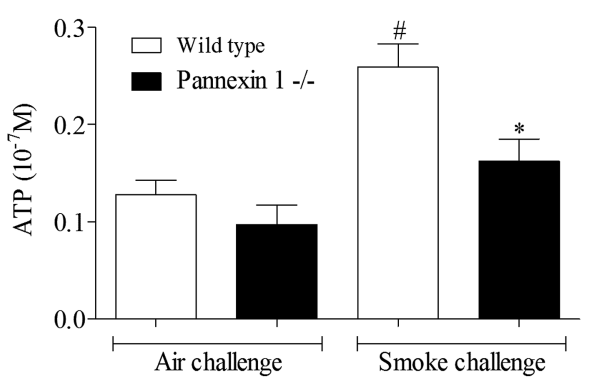

B BALF IL-1 $\beta$

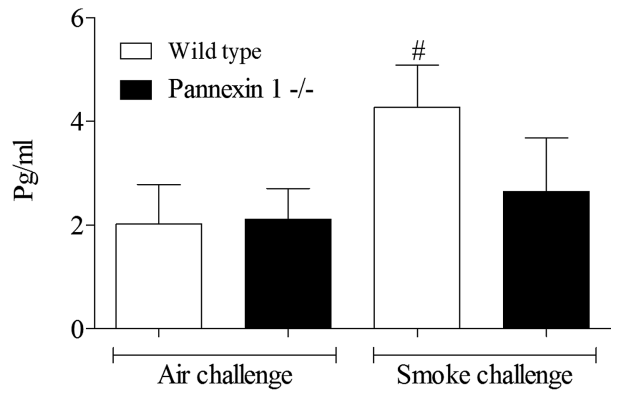

C BALF Neutrophilia

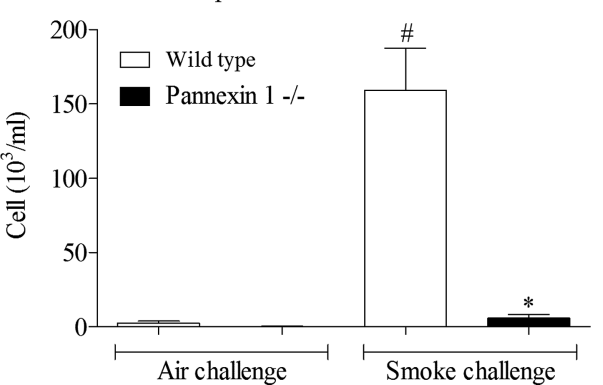

Figure 8 The role of pannexin-1 in acute cigarette smoking (CS)-induced murine airway inflammation. Pannexin-1 ${ }^{-1-}$ mice were exposed to CS or room air (control) twice daily for 3 consecutive days alongside wild-type controls. Bronchoalveolar lavage fluid (BALF) was collected $24 \mathrm{~h}$ after the last exposure for measurement of neutrophil (C), interleukin (IL)-1 $\beta$ (B) and ATP (A) levels. Data are represented as mean $\pm S E M$ for $n=8$ animals in each group. Statistical significance was determined using Mann-Whitney $U$ test. $\# p<0.05$, denoting a significant difference between the smoke-exposed and air-exposed wild-type groups; ${ }^{*} p<0.05$, denoting a significant difference between the smoke-exposed knock-outs and wild-types.

analysis of the CSM would suggest otherwise. Furthermore, changes in osmolarity, a known trigger of TRPV4 activation, ${ }^{27}$ seems an unlikely scenario in this model system. Therefore, it would seem that either constituents in CSM directly trigger the channels or they result in the production of a channel modulator. Indeed, we are currently exploring ways of identifying likely candidates from the $4000+$ chemicals reported to be present in CS. It is

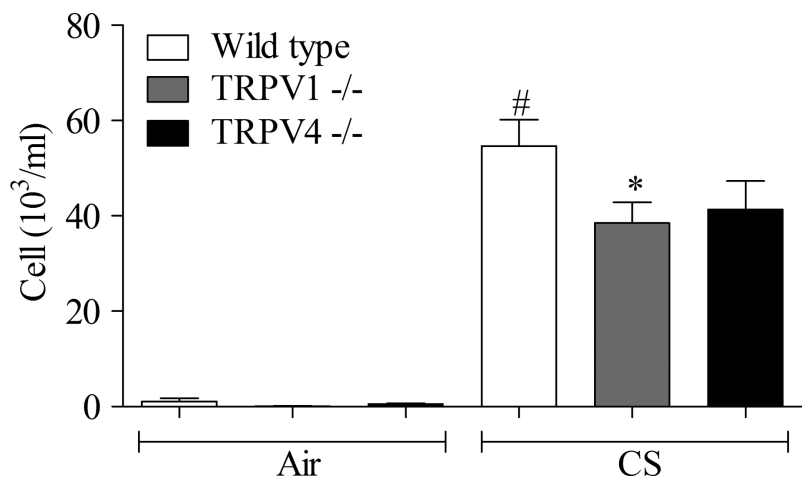

Figure 9 The role of TRPV1 and V4 in subchronic cigarette smoking (CS)-induced murine airway inflammation. Trpv1 $1^{-l-}$ and Trpv $4^{-l-}$ mice were exposed to CS or room air (control) twice daily for 14 consecutive days alongside wild-type controls. Bronchoalveolar lavage fluid was collected $24 \mathrm{~h}$ after the last exposure for measurement of neutrophils. Data are represented as mean \pm SEM for $n=8$ animals in each group. Statistical significance was determined using analysis of variance with a Bonferroni post-test; \#p<0.05, denoting a significant difference between the smoke-exposed and air-exposed wild-type groups; ${ }^{*} p<0.05$, denoting a significant difference between the smoke-exposed knock-outs and wild-types. also currently not clear how activation of TRP channels triggers the opening of the pannexin-1 pore. The most likely mechanism would simply be that increases in intracellular $\mathrm{Ca}^{2+}$ and membrane depolarisation are sufficient or that a second messenger signal is required. ${ }^{18}$ However, the fact that the pannexin- 1 pore is central to the response does mirror observations made by others in different biological systems. ${ }^{22-24} 2627$ The time course of ATP release in vitro is intriguing, it would appear that the release is a not a rapid process (taking a few hours to reach a plateau), suggesting that either the process is slow or that perhaps the CS induction of the possible TRP channel activators takes time. Furthermore, the data would suggest that the cells stop releasing ATP after a time or that enzymes which cleave it are induced.

Finally, it is unclear why ATP is still detectable, especially in vivo, as one would assume that under normal circumstances ATP would be cleaved rapidly. Indeed, preliminary studies with exogenous ATP added to BALF from smoke-exposed mice showed that it is undetectable with the specific ATP assay used. This suggests that the ecto-ATPases that breakdown ATP are functional in the CS-exposed mouse lung and perhaps the ATP measured in the BALF by our group and others is somehow protected from enzymatic activity, and this observation warrants further investigation.

In summary, this data represents a significant breakthrough in understanding the mechanism by which CS exposure leads to the release of ATP. Furthermore, preliminary expression data in clinical samples appears to validate the preclinical findings. We, therefore, propose that pharmacological modulation of the TRPV1/4-pannexin-1 axis may provide clinical benefit to patients with COPD by attenuating the dysregulated, steroid-resistant inflammation which is associated with the decline in lungfunction seen in patients with COPD. 
A TRPV1

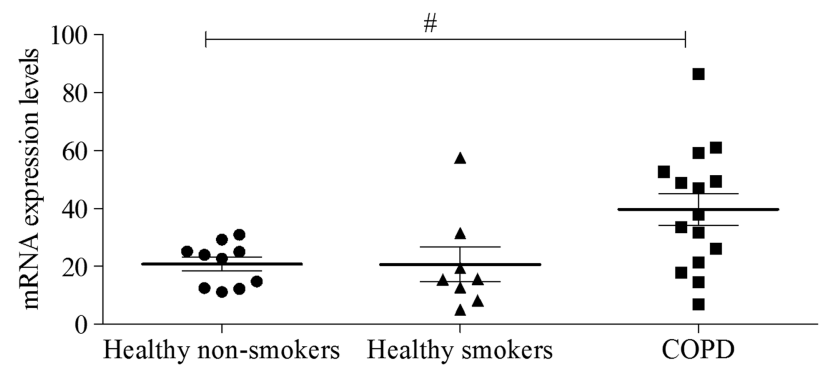

C TRPV3

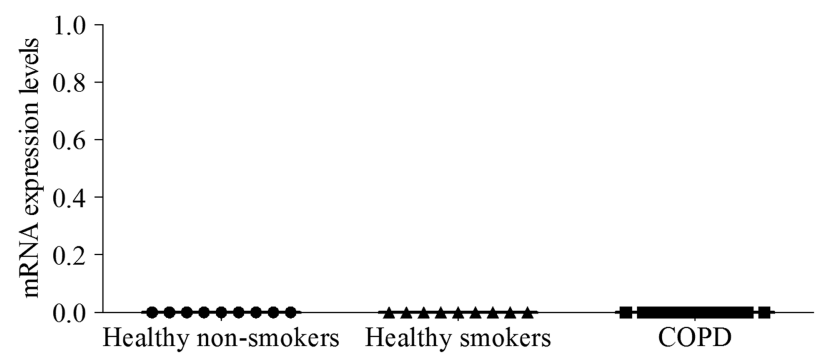

B TRPV2

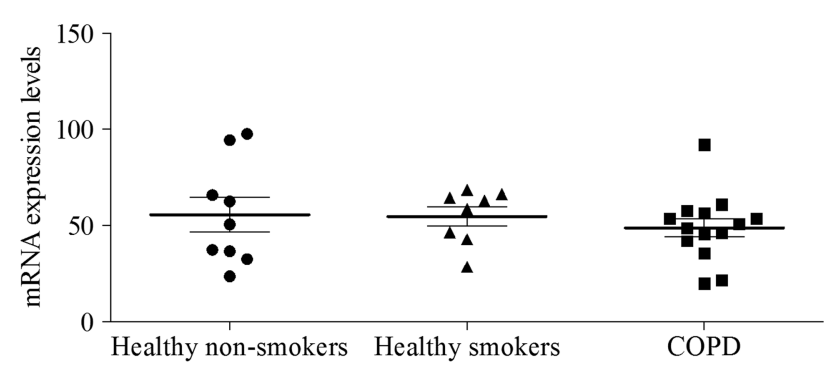

D TRPV4

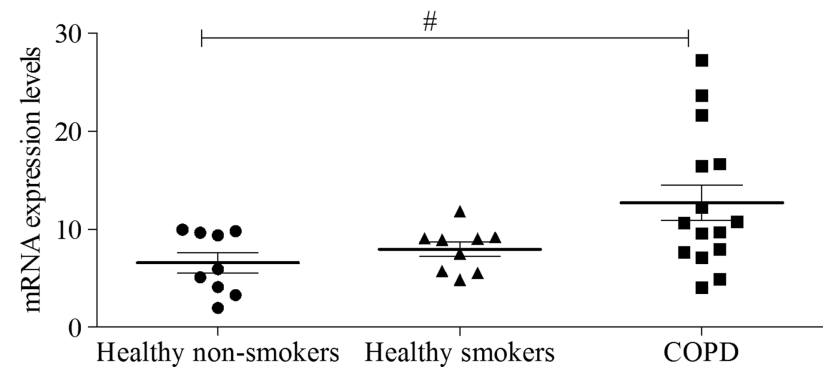

Figure 10 Transient receptor potential (TRP) channel expression in human lung parenchyma. Human lung parenchymal samples were collected from healthy non-smokers, healthy smokers and patients with COPD. RNA was extracted and reverse transcribed to CDNA before measurement of TRPV1 (A), TRPV2 (B), TRPV3 (C) and TRPV4 (D) mRNA expression levels using TaqMan reagents and protocols. Data are expressed as mean \pm SEM expression levels relative to internal control (18S); $\# p<0.05$, denotes a significant difference between healthy smokers and patients with COPD, determined by one-way analysis of variance followed by Dunn's multiple comparisons posthoc test.

Acknowledgements $P 2 \times 7^{-/-}$mice were kindly supplied by Professor Jean Kanellopoulos, Université Paris-Sud, France, Trpv $4^{-1}$ mice were kindly supplied by Riken Bioresource Centre, and Dr Vishva Dixit from Genentech generously provided the pannexin $1^{-\frac{1}{m}}$ mice.

Contributors Conception and design: MB, MGB, MAB. Data generation, analysis and interpretation: MB, SE, BD, LY-B, EDD, SAM. Drafting the manuscript for important intellectual content: MB, MGB, MAB. All authors reviewed the manuscript and approved the final draft

Competing interests MB was supported by an NHLI COPD PhD studentship, SE was supported by a postdoctoral award from Roche Pharmaceuticals, Nutley, USA. LY-B was funded by a Capacity Building Award in Integrative Mammalian Biology funded by the BBSRC, BPS, HEFCE, KTN \& MRC. EDD was funded by a Wellcome Trust project grant (089301/Z/09/Z) and Boehringer-Ingelheim. SAM and MAB were funded by project grants from the MRC, UK (G0800195 and G0800196, respectively). The human tissue experiments in this study were undertaken with the support of the NIHR Respiratory Disease Biomedical Research Unit at the Royal Brompton and Harefield NHS Foundation Trust and Imperial College London.

Provenance and peer review Not commissioned; externally peer reviewed.

\section{REFERENCES}

1 Silverman E, Speizer F. Risk factors for the development of chronic obstructive pulmonary disease. Med Clin North Am 1996;80:501-22.

2 Vestbo J, Hurd SS, Agusti AG, et al. Global strategy for the diagnosis, management, and prevention of chronic obstructive pulmonary disease. Am J Respir Crit Care Med 2013;187:347-65.

3 Lopez AD, Murray CC. The global burden of disease. Nat Med 1998:4:1241-3.

4 Di Stefano A, Turato G, Maestrelli P, et al. Airflow limitation in chronic bronchitis is associated with T-lymphocyte and macrophage infiltration of the bronchial mucosa. Am J Respir Crit Care Med 1996;153:629-32.

5 Saetta M, Turato G, Facchini FM, et al. Inflammatory cells in the bronchial glands of smokers with chronic bronchitis. Am J Respir Crit Care Med 1997;156:1633-9.

6 Sapey E, Ahmad A, Bayley D, et al. Imbalances between interleukin-1 and tumor necrosis factor agonists and antagonists in stable COPD. J Clin Immunol 2009;29:508-16.

7 Rovina N, Dima E, Gerassimou C, et al. Interleukin-18 in induced sputum: association with lung function in chronic obstructive pulmonary disease. Respir Med 2009;103:1056-62.

8 Perregaux D, Gabel CA. Interleukin-1 beta maturation and release in response to ATP and nigericin. Evidence that potassium depletion mediated by these agents is a necessary and common feature of their activity. J Biol Chem 1994;269:15195-203.

9 Mariathasan S, Monack DM. Inflammasome adaptors and sensors: intracellular regulators of infection and inflammation. Nat Rev Immunol 2007;7:31-40.

10 Qu Y, Franchi L, Nunez G, et al. Nonclassical IL-1 beta secretion stimulated by P2X7 receptors is dependent on inflammasome activation and correlated with exosome release in murine macrophages. J Immunol 2007;179:1913-25.

11 Mortaz E, Braber S, Nazary M, et al. ATP in the pathogenesis of lung emphysema. Eur J Pharmacol 2009;619:92-6.

12 Lommatzsch M, Cicko S, Müller T, et al. Extracellular adenosine triphosphate and chronic obstructive pulmonary disease. Am J Respir Crit Care Med 2010;181:928-34.

13 Mohsenin A, Blackburn M. Adenosine signaling in asthma and chronic obstructive pulmonary disease. Curr Opin Pulm Med 2006;12:54-9.

14 Cicko S, Lucattelli M, Müller $T$, et al. Purinergic receptor inhibition prevents the development of smoke-induced lung injury and emphysema. J Immunol 2010;185:688-97.

15 Eltom S, Stevenson CS, Rastrick J, et al. P2X7 receptor and caspase 1 activation are central to airway inflammation observed after exposure to tobacco smoke. PLoS ONE 2011;6:e24097.

16 Lucattelli M, Cicko S, Müller T, et al. P2X7 receptor signaling in the pathogenesis of smoke-induced lung inflammation and emphysema. Am J Respir Cell Mol Biol 2011:44:423-9.

17 Churg A, Zhou S, Wang $X$, et al. The role of interleukin-1beta in murine cigarette smoke-induced emphysema and small airway remodeling. Am J Respir Cell Mol Biol 2009:40:482-90.

18 Praetorius HA, Leipziger J. ATP release from non-excitable cells. Purinergic Signal 2009;5:433-46.

19 Lazarowski ER, Boucher RC, Harden TK. Mechanisms of release of nucleotides and integration of their action as P2X-and P2Y-receptor activating molecules. Mol Pharmacol 2003;64:785-95.

20 Junger WG. Immune cell regulation by autocrine purinergic signalling. Nat Rev Immunol 2011;11:201-12.

21 Baroja-Mazo A, Barberà-Cremades M, Pelegrín P. The participation of plasma membrane hemichannels to purinergic signaling. Biochim Biophys Acta 2013;1828:79-93.

22 Qu Y, Misaghi S, Newton K, et al. Pannexin-1 is required for ATP release during apoptosis but not for inflammasome activation. J Immunol 2011;186:6553-61.

23 Lemaire I, Falzoni S, Zhang B, et al. The P2X7 receptor and Pannexin-1 are both required for the promotion of multinucleated macrophages by the inflammatory cytokine GM-CSF. J Immunol 2011;187:3878-87. 
24 Ransford GA, Fregien N, Qiu F, et al. Pannexin 1 contributes to ATP release in airway epithelia. Am J Respir Cell Mol Biol 2009:41:525-34.

25 Karlberg M, Ekoff M, Huang DCS, et al. The BH3-mimetic ABT-737 induces mast cell apoptosis in vitro and in vivo: potential for therapeutics. J Immunol 2010;185:2555-62.

26 Huang Y-J, Maruyama Y, Dvoryanchikov G, et al. The role of pannexin 1 hemichannels in ATP release and cell-cell communication in mouse taste buds. Proc Natl Acad Sci USA 2007:104:6436-41.

27 Seminario-Vidal L, Okada SF, Sesma Jl, et al. Rho signaling regulates pannexin 1-mediated ATP release from airway epithelia. J Biol Chem 2011:286:26277-86.

28 Voets T, Nilius B. TRPs make sense. J Membr Biol 2003;192:1-8.

29 Alvarez DF, King JA, Weber D, et al. Transient receptor potential vanilloid 4-mediated disruption of the alveolar septal barrier: a novel mechanism of acute lung injury. Circ Res 2006;99:988-95.

30 Willette RN, Bao W, Nerurkar S, et al. Systemic activation of the transient receptor potential vanilloid subtype 4 channel causes endothelial failure and circulatory collapse: part 2. J Pharmacol Exp Ther 2008;326: $443-52$

31 MacNee W, Rennard S, Hunt J. Evaluation of exhaled breath condensate $\mathrm{pH}$ as a biomarker for COPD. Respir Med 2011:105:1037-45.

32 Nassini R, Pedretti P, Moretto N, et al. Transient receptor potential ankyrin 1 channel localized to non-neuronal airway cells promotes non-neurogenic inflammation. PLOS ONE 2012:7:e42454.
33 Andrè E, Campi B, Materazzi S. Cigarette smoke-induced neurogenic inflammation is mediated by $\alpha, \beta$-unsaturated aldehydes and the TRPA 1 receptor in rodents. J Clin Invest 2008;118:2574-82.

34 Birrell MA, Wong S, Catley MC, et al. Impact of tobacco-smoke on key signaling pathways in the innate immune response in lung macrophages. J Cell Physiol 2008:214:27-37.

35 Grace M, Birrell M, Dubuis E, et al. Transient receptor potential channels mediate the tussive response to prostaglandin E2 and bradykinin. Thorax 2012;67:891-900.

36 Wong S, Belvisi MG, Birrell MA. MMP/TIMP expression profiles in distinct lung disease models: implications for possible future therapies. Respir Res 2009;10:72.

37 Mizuno A, Matsumoto N, Imai M, et al. Impaired osmotic sensation in mice lacking TRPV4. Am J Physiol Cell Physiol 2003;285:C96-101.

38 Suzuki M, Mizuno A, Kodaira K, et al. Impaired pressure sensation in mice lacking TRPV4. J Biol Chem 2003;278:22664-8.

39 Sethi JM, Rochester CL. Smoking and chronic obstructive pulmonary disease. Clin Chest Med 2000;21:67-86, viii.

40 Lappalainen U, Whitsett JA, Wert SE, et al. Interleukin-1 beta causes pulmonary inflammation, emphysema, and airway remodeling in the adult murine lung. Am J Respir Cell Mol Biol 2005:32:311-18.

41 Zhu G, Gulsvik A, Bakke P, et al. Association of TRPV4 gene polymorphisms with chronic obstructive pulmonary disease. Hum Mol Genet 2009;18:2053-62.

42 Obeidat M, Wain LV, Shrine N, et al. A comprehensive evaluation of potential lung function associated genes in the SpiroMeta general population sample. PLOS ONE 2011:6:e19382 